sixtieth part of a day's destruction in the Formby Channel, it is a wonder that there are any left.

A point emphasized in the review, but seemingly missed by Mr. Walker, is that the young fish are always promptly returned to the sea by the Solway shrimpers, and the fact that the industry flourishes in spite of the delay so caused shows that the destruction which ensues from the practices described by $\mathrm{Mr}$. Walker is quite unnecessary. Dr. Fulton has experimentally proved that the proportion of young flat-fish of a certain size (say above an inch) that would not survive if returned to the sea is small, so that it is evident that Mr. Ascroft's "axiom that 90 per cent. of fish that come on board a boat is destroyed " holds good from no fault of the trawl itself, but simply from a discreditable carelessness on the part of the man.

Mr. Walker's experiences at the mouth of the Dee show that the shrimps and the young soles (species?) have different habitats in that river, so that his suggestions as to the limitation of shrimp-trawling seem rather superfluous, since it may be supposed that the trawler would fish where he knew he could get shrimps, and not go out of his way to catch what he did not want. I have noticed myself on the west coast of Ireland that the minute post-larval flat-fisb, smaller than those dealt with by Fulton, and which are undoubtedly killed by the meshes of the shrimp-trawl, were never taken on ground frequented by shrimps, where, indeed, as one may judge from the relations of the two forms in captivity, the weaker would have a poor chance of surviving.

Everyone will agree with Mr. Walker that it is most necessary to ascertain the habitat of the young fish at different times of the year, and to this end the energies of the Marine Biological Association in England, the Fishery Board in Scotland, and the Royal Dublin Society in Ireland, have been for some time directed ; and the assistance that might be rendered by a series of observations by one possessing the experience and opportunities of Mr. Walker would be incalculable. Until, however, our knowledge on the subject is much more complete, I question the advantage of strewing boulders about the bottom of the sea. Even if they remained to accomplish their purpose of interfering with trawling, there is the danger that they would form an attractive shelter, not to the young flat-fish that stand in no need of it, but to some of their natural enemies.

Dublin, December 27, I891.

ERnest W. L. Holt.

\section{A New Precessional Globe.}

To facilitate the understanding of the effects of precession, I have made a new arrangement of the celestial globe. A globe mounted in the new way can give a representation of the starry heavens for every place on the earth, and for any date, both past and future.

The globe is fastened in a ring, so that it can be turned round an axis that goes through the poles of the ecliptic, but can also be fixed in any position by a pair of screws. The amount of turning is to be measured by a divided circle.

The ring above mentioned-which we will call ring I.-is movable in another ring (ring II.), round an axis, which forms a right angle with the axis formerly mentioned. The inclination between ring $\mathrm{I}$. and ring II. can be measured by an index; it must equal the obliquity of the ecliptic.

Ring II. is fastened finally in a third and extreme ring (ring III.), so that it can be turned round an axis which forms an angle of $90^{\circ}$ with the axis of ring II. Ring III. is mounted on a stand with a horizon-circle, so that its axis can be inclined at pleasure to the plane of the horizon-circle. The inclination may be read on a scale engraved on ring III.

To adjust the apparatus to show the firmament at any appointed place and time, one must place ring III. so that its inclination towards the horizon-circle equals the latitude of the place. Then ring II. must be turned so that its plane coincides with the plane of ring III. The angle between I. and II. must be equal to the obliquity of the ecliptic at the appointed time. Finaliy, the globe must be turned round the axis which goes through the poles of the ecliptic, till the point of the heaven, which is the celestial pole for the time appointed, comes under the ax.s round which ring II. turns in ring III. If the globe is then fastened in ring I., and ring I. in ring II., with screws, by turning the globe in ring III. one can see at a glance which stars are setting and rising, and which are always above the horizon.

By making Vega, for example, the celestial pole (I4,000 A.D.), one can see immediately that for the latitude of London at that remote period, the Cross would be seen at the southern horizon, and that Sirius then did not rise at all.

K. HAAS.

Vienna.

\section{Simple Proof of Euclid II. 9 and ro.}

IN NATURE of December 24 (p. I89) a simple proof of Euclid II. 9 and Io is given, which it is stated is believed to be new. It may therefore be of interest to your readers to know that these proofs are given in an edition of Euclid which we have now in the press. As the author, Mr. Brent, is resident at Dunedin, New Zealand, we are unable to state whether he lays claim or not to any originality in respect to them: in any case, as he has been engaged in mathematical teaching for many years, these and similar proofs of other propositions in Euclid II. have clearly been more widely employed than has been supposed.

34 King Street, Covent Garden, London, January 4.

\section{THE ALLEGED DISCOVERY OF A BACILLUS IN INFLUENZA.}

FROM the behaviour of influenza as an epidemic, it seems not unreasonable to suppose that it may have as its cause a living and multiplying organism; and when influenza reappeared, after an interval of many years, in the latter part of I889, and more especially since its communicability from person to person, formerly disputed, has come to be generally admitted, the public mind, medical and lay, has been in expectation of the announcement that a specific microbe had been discovered as the cause of the disease.

Even in diseases, however, of which the characters point most strongly to a parasitic microbe as their cause, the discovery of such an organism is by no means an easy matter. Thus, no micro-oryanism has as yet been identified as the cause of small-pox, although this disease is always more or less with us; breeds true ; has distinct characters, and a definite localization on the skin; and propagates by a contagion which retains its activity for very long periods - circumstances which point to a specific organism as its cause, and might be thought to facilitate its discovery.

From a priori considerations we must suppose the properties of the hypothetical influenza microbe to be as follows. The diffusibility of the poison through the air shows that it must be very minute and readily suspended. For the same reason it must belong to the class of aërobic organisms, $i e$. those for whose existence oxygen is necessary, or at any rate not hurtful. It must multiply with extreme rapidity. It must be capable of multiplying in the bodies, or secretions, of human beings; and probably also in some medium or media outside the human body-perhaps on damp ground-surfaces, or in confined air laden with dust and organic matter. One can hardly suppose it capable of multiplying in pure air, as it would lack pabulum; perhaps, as Dr. Symes Thompson suggests, particles of organic dust floating in the air may serve as rafts for it to live on. As, however, influenza prevails under the most opposite conditions of season, climate, and weather, our supposed microbe, if it can live in the air, must be able to flourish under a great range of temperatures and degrees of humidity. I am not aware of any instances of long retention of contagion, such as would lead us to postulate the possession by our microbe of resting spores. From these considerations we might have expected that it would be more likely to turn out to be a micrococcus than a bacillus.

From what is known of the pathology of some other diseases of microbic origin, as tetanus and diphtheria, it seems possible that the immediate cause of the symptoms of influenza may be the presence in the blood and tissues, not of the microbe itself, but of the poison NO. I I 59, VOL. 45] 
manufactured by it. The microbe itself may disappear early in the case ; but the poison formed by it pervades the whole body, and especially the nervous system, and produces profound and lasting effects. An early disappearance of the microbe in influenza would explain the failure to find it on post-mortem examination ; death from influenza being usually the result of complications rather than of the primary disease. Influenza is infectious at an early stage of the disease ; but it is not known how long the infectious condition may last : some cases point to its being infectious as late as the eighth day, and we must suppose that, as long as the disease is communicable, the microbe retains its vitality.

Evidently there is a power of resistance in the human body to the invasion of the microbe, varying in different persons, for not all who are exposed to the infection contract the disease ; the resistance to it being weakened by depressing influences-as fatigue, and exposure to changes of temperature.

The resistance to the poison seems also to be overcome when the dose is large: a certain degree of concentration seems to be necessary in order for the disease to take on an epidemic form.

The pabulum for the microbe, or the number of susceptible persons, seems also to be soon exhausted; for the decline of an epidemic as regards the number of new cases is often almost as rapid as its increase. At the same time the immunity conferred by an attack of influenza is of short duration, for a person may suffer repeatedly from the disease; and the same holds as regards communities, for many localities have suffered repeated epidemics of influenza during the last few years; whereas an epidemic of one of the ordinary infectious diseases is usually succeeded by a long period of comparative freedom. These are some of the points which have to be taken account of in any theory of the causation of influenza.

During the past week the announcement has been made in the public press of the discovery by Dr. Pfeiffer, in the Royal Institute for Infectious Diseases at Berlin, of a bacillus which he looks upon as the cause of influenza.

It will be remembered that a similar announcement, made, in January I 890 , by Dr. Jölles, of Vienna, was not confirmed. Since then various observers have discovered in the sputa and lungs of influenza patients, microorganisms of one kind or another; but their statements are conflicting, and the forms met with are some of them at least known to occur in other diseases; so that the inference is that they were either accidentally present, or connected with secondary affections for which the attack of influenza had prepared the way. Whether Dr. Pfeiffer's discovery will be more successful in gaining acceptance remains to be seen; but the position of its author, and the alleged confirmation of his results by Dr. Koch, will no doubt secure for it a respectful consideration.

As Dr. Koch has pointed out, in order that the relation of a particular micro-organism to a particular disease, as cause and effect, may be considered satisfactorily proved, the following conditions must be complied with :-

(I) The micro-organism in question must be present in the secretions, blood, or diseased tissues of the subject of the disease.

(2) The micro-organisms in question must be isolated and cultivated-all other organisms being excluded-in suitable media outside the animal body, through several generations of cultures.

(3) The micro-organisms, thus cultivated, when introduced into the body of a healthy animal of a susceptible kind must be capable of producing in it the disease in question.

(4) In the animal in which the disease has thus been produced the same micro-organism must again be found.

It is stated that, for the investigation of the etiology of influenza, a clinique for influenza patients was opened in
September last in the Royal Institute for Infectious Diseases, and that the result of the exhaustive examination of the cases was the discovery in the matter discharged from the patients' lungs of a bacillus found in no other cases of disease of the respiratory organs, and which, as the patient recovered, gradually disappeared. It is stated that the bacilli were cultivated to the fifth generation, and that, inoculated into monkeys and rabbits, they produced in every case the symptoms of influenza.

It is added that these results were confirmed by Dr. Koch, who further discovered the same bacillus in the blood of patients in the febrile stage of influenza.

An account of the discovery is promised in the Deutsche medicinische Wochenschrift, and will be awaited with interest.

H. F. P.

\section{ON THE MATTER THROWN UP DURING THE SUBMARINE ERUPTION NORTH-WEST OF PANTELLERIA, OCTOBER I89I.}

WE did not reach Pantelleria till November 5, ten days after the end of the eruption, but a certain number of specimens had been secured at the time by people who went out in boats. Dr. Errera, who helped us in other ways, kindly gave me some pieces of a bomb from his own collection; and others, among them a goodsized bomb, some 30 inches in its longest diameter, were obtained from the inhabitants. Out of a number of small pieces from different people, I did not see any that might not have formed some part of a mass such as this last, and I have no evidence that any other kind of material was erupted.

\section{General, Structural, and Mineralogical Characters.}

Riccò (Comptes rendus, November 25, 1891) says that some of the bombs had a diameter of 2 metres, and that the prevailing shape was an "ellipsoid of revolution." They were not only porous in texture, but contained large cavities, and floated for a time, but pieces taken separately are fairly heavy. Riccò mentions a specific gravity of $\mathrm{I}_{4}$ (perhaps for a bomb when unbroken). A 4-ounce piece of the coarser material from the inside displaced less than half its weight of water, giving me specific gravity $2 \% 3$.

What most struck the possessors of specimens was their coaly blackness. Nevertheless, there was on the outside of the bombs a distinctly brownish layer, an inch perhaps in thickness, which the eye, or better, the pocket lens, shows to be due to vesiculation of the transparent brown glass that here forms the ground mass In certain places vesiculation has been carried so far tha we have a coarse" type of the "thread lace scoria," de scribed among others by Dana, from Hawaii (Amer Journ. Sci., March 1888, p. 213 , or his book, "Character istics of Volcanoes," p. 163, I 890).

In the brown glass of this part sections show numerous narrow crystals of triclinic felspar, and in places olivine and magnetite, and probably a little augite.

Beneath this brownish layer may occur another, say half an inch thick, coarser and darker than the former; but which in sections can still be seen to be for the most part brown transparent glass, with the above-mentioned minerals in it.

We are thus led to a layer perhaps an inch or twc thick, ${ }^{2}$ coarsely spongy, black, of pitchstone lustre, whick

2 I find a prettier example of this structure on one side of a piece of scori picked up last month near the base of the active cone of Vesuvius. It wa partly covered by the brown dust ejected since the tappiug of the lava on the side of the Atrio del Cavallo last summer. I do not know, therefore when it was erupted. I find on this, as on scoria that I saw erupted October I889, a tendency to form Pele's hair (on a very sinall scale).

Unfortunately, the only whole bomb that I saw went to pieces on the way to England. The point worth noticing is the difference in structureo different parts; and I give these rough measurements, taken from piece in my posses sion, merely to show the kind of dimensions with which we dealing.

NO. I I 59, VOL. 45] 